\title{
The hot dayside and asymmetric transit of WASP-189 b seen by CHEOPS ${ }^{\star}$
}

\author{
M. Lendl ${ }^{1,2}$, Sz. Csizmadia ${ }^{3}$, A. Deline ${ }^{1}$, L. Fossati², D. Kitzmann ${ }^{4}$, K. Heng ${ }^{4}$, S. Hoyer ${ }^{5}$, S. Salmon ${ }^{1,7}$, W. Benz ${ }^{6,4}$,
} C. Broeg ${ }^{6}$, D. Ehrenreich ${ }^{1}$, A. Fortier ${ }^{6}$, D. Queloz ${ }^{1,8}$, A. Bonfanti ${ }^{2}$, A. Brandeker ${ }^{10}$, A. Collier Cameron ${ }^{11}$, L. Delrez ${ }^{7,12,1}$, A. Garcia Muñoz ${ }^{13}$, M. J. Hooton ${ }^{6}$, P. F. L. Maxted ${ }^{9}$, B. M. Morris ${ }^{4}$, V. Van Grootel ${ }^{7}$, T. G. Wilson ${ }^{11}$, Y. Alibert ${ }^{6}$, R. Alonso ${ }^{14,15}$, J. Asquier ${ }^{16}$, T. Bandy ${ }^{6}$, T. Bárczy ${ }^{17}$, D. Barrado ${ }^{18}$, S. C. C. Barros ${ }^{19,20}$, W. Baumjohann ${ }^{2}$, M. Beck ${ }^{1}$, T. Beck ${ }^{4}$, A. Bekkelien ${ }^{1}$, M. Bergomi ${ }^{21}$, N. Billot ${ }^{1}$, F. Biondi ${ }^{21}$, X. Bonfils ${ }^{22}$, V. Bourrier ${ }^{1}$, M.-D. Busch ${ }^{6}$, J. Cabrera ${ }^{3}$, V. Cessa ${ }^{4}$, S. Charnoz ${ }^{23}$, B. Chazelas ${ }^{1}$, C. Corral Van Damme ${ }^{16}$, M. B. Davies ${ }^{24}$, M. Deleuil ${ }^{5}$, O. D. S. Demangeon ${ }^{5,19}$, B.-O. Demory ${ }^{4}$, A. Erikson ${ }^{3}$, J. Farinato ${ }^{21}$, M. Fridlund ${ }^{25,26}$, D. Futyan ${ }^{1}$, D. Gandolfi2 ${ }^{27}$, M. Gillon ${ }^{12}$, P. Guterman ${ }^{5,28}$, J. Hasiba ${ }^{2}$, E. Hernandez ${ }^{4}$, K. G. Isaak ${ }^{16}$, L. Kiss ${ }^{29}$, T. Kuntzer ${ }^{1}$, A. Lecavelier des Etangs ${ }^{30}$, T. Lüftinger ${ }^{31}$, J. Laskar ${ }^{32}$, C. Lovis ${ }^{1}$, D. Magrin ${ }^{21}$, L. Malvasio ${ }^{4}$, L. Marafatto ${ }^{21}$, H. Michaelis ${ }^{3}$, M. Munari ${ }^{33}$, V. Nascimbeni ${ }^{21}$, G. Olofsson ${ }^{10}$, H. Ottacher ${ }^{2}$, R. Ottensamer ${ }^{31}$, I. Pagano ${ }^{33}$, E. Palle ${ }^{14,15}$, G. Peter ${ }^{34}$, D. Piazza ${ }^{6}$, G. Piotto ${ }^{35,21}$, D. Pollacco ${ }^{36}$, F. Ratti ${ }^{16}$, H. Rauer ${ }^{3,13,37}$, R. Ragazzoni ${ }^{21}$, N. Rando ${ }^{16}$, I. Ribas $^{38,39}$, M. Rieder ${ }^{6}$, R. Rohlfs ${ }^{1}$, F. Safa ${ }^{16}$, N. C. Santos ${ }^{19,20}$, G. Scandariato ${ }^{33}$, D. Ségransan ${ }^{1}$, A. E. Simon ${ }^{6}$, V. Singh $^{33}$, A. M. S. Smith ${ }^{3}$, M. Sordet ${ }^{1}$, S. G. Sousa ${ }^{19}$, M. Steller ${ }^{2}$, Gy. M. Szabó ${ }^{40,41}$, N. Thomas ${ }^{6}$, M. Tschentscher ${ }^{3}$, S. Udry $^{1}$, V. Viotto ${ }^{21}$, I. Walter ${ }^{34}$, N. A. Walton ${ }^{42}$, F. Wildi ${ }^{1}$, and D. Wolter ${ }^{3}$

(Affiliations can be found after the references)

Received 16 June 2020 / Accepted 17 September 2020

\begin{abstract}
The CHEOPS space mission dedicated to exoplanet follow-up was launched in December 2019, equipped with the capacity to perform photometric measurements at the $20 \mathrm{ppm}$ level. As CHEOPS carries out its observations in a broad optical passband, it can provide insights into the reflected light from exoplanets and constrain the short-wavelength thermal emission for the hottest of planets by observing occultations and phase curves. Here, we report the first CHEOPS observation of an occultation, namely, that of the hot Jupiter WASP-189 b, a $M_{\mathrm{P}} \approx 2 M_{\mathrm{J}}$ planet orbiting an A-type star. We detected the occultation of WASP-189 b at high significance in individual measurements and derived an occultation depth of $\mathrm{dF}=87.9 \pm 4.3 \mathrm{ppm}$ based on four occultations. We compared these measurements to model predictions and we find that they are consistent with an unreflective atmosphere heated to a temperature of $3435 \pm 27 \mathrm{~K}$, when assuming inefficient heat redistribution. Furthermore, we present two transits of WASP-189 b observed by CHEOPS. These transits have an asymmetric shape that we attribute to gravity darkening of the host star caused by its high rotation rate. We used these measurements to refine the planetary parameters, finding a $\sim 25 \%$ deeper transit compared to the discovery paper and updating the radius of WASP- $189 \mathrm{~b}$ to $1.619 \pm 0.021 R_{\mathrm{J}}$. We further measured the projected orbital obliquity to be $\lambda=86.4_{-4.4}^{+2.9^{\circ}}$, a value that is in good agreement with a previous measurement from spectroscopic observations, and derived a true obliquity of $\Psi=85.4 \pm 4.3^{\circ}$. Finally, we provide reference values for the photometric precision attained by the CHEOPS satellite: for the $V=6.6 \mathrm{mag}$ star, and using a 1-h binning, we obtain a residual RMS between 10 and $17 \mathrm{ppm}$ on the individual light curves, and 5.7 ppm when combining the four visits.
\end{abstract}

Key words. techniques: photometric - planets and satellites: atmospheres - planets and satellites: individual: WASP-189b

\section{Introduction}

The Characterising Exoplanets Satellite (CHEOPS) is the first European space mission dedicated primarily to the study of known extrasolar planets. It consists of a $30 \mathrm{~cm}$ (effective) aperture telescope collecting ultra-high precision time-series photometry of exoplanetary systems in a broad optical passband (Benz et al. 2020). Unlike the previous space observatories dedicated to exoplanets, CoRoT (Baglin et al. 2006), Kepler (Borucki et al. 2010), K2 (Howell et al. 2014), and the ongoing TESS

\footnotetext{
* The photometric time series data are only available at the CDS via anonymous ftp to cdsarc.u-strasbg. fr $(130.79 .128 .5)$ or via http://cdsarc.u-strasbg.fr/viz-bin/cat/J/A+A/643/A94
}

mission (Ricker et al. 2014), CHEOPS is a pointed mission, optimised to obtain high-cadence photometric observations at the $20 \mathrm{ppm}$ level for a single star at a time. CHEOPS was launched successfully into a $700 \mathrm{~km}$ altitude Sun-synchronous polar orbit on 18 December 2019 and its first science observations were obtained in late March 2020.

As one of its first scientific targets, CHEOPS observed the ultra-hot Jupiter WASP-189 b (Anderson et al. 2018), a gas giant transiting the bright $(V=6.6 \mathrm{mag})$ A-type star HD 133112 . WASP-189 $\mathrm{b}$ is one of the most highly irradiated planets known thus far, with a dayside equilibrium temperature of $\sim 3400 \mathrm{~K}$ (Anderson et al. 2018). It orbits an early-type star similarly to the extreme object KELT-9b (Gaudi et al. 2017), but with a longer orbital period of 2.7 days, placing it closer, in temperature, to 



Fig. 1. Individual CHEOPS observations of four WASP-189 b occultations. In both panels, visits are shown chronologically from top to bottom, occurring on 19, 27, and 30 March and 7 April 2020, respectively. Left: uncorrected observations (black points) together with their full (baseline and occultation, red line) light-curve models. Blue vertical dash-dotted lines indicate the beginning and end of occultation. Right: data (black points) corrected for the instrumental and stellar trends, together with the occultation model (red line).

ultra-short period planets orbiting $\mathrm{F}$ and $\mathrm{G}$ stars. As such, this object allows us to comparatively probe the impact of different stellar spectral energy distributions and, in particular, strong short-wavelength irradiation on planetary atmospheres. As it is orbiting around an A-type star, the system is also relatively young $(730 \pm 130 \mathrm{Myr}$, see Sect. 2.2), providing us with a window into the atmospheric evolution of close-in gas giants.

In this paper, we report on CHEOPS observations of four occultations and two transits of WASP-189 b. We use the occultations to constrain the planet's temperature and reflective properties and the transits to revise the planetary radius and determine the system's orbital obliquity from the gravity darkening of the host star and the associated light curve asymmetry. We describe the observations and data reduction in Sect. 2, discuss the results in Sect. 3, and present a brief conclusion in Sect. 4.

\section{Observations, data reduction, and analysis}

\subsection{CHEOPS observations of WASP-189 b}

We observed four occultations of WASP-189b between 19 March and 7 April 2020. The individual observations lasted between 12.4 and $13 \mathrm{~h}$, distributed over either seven or eight spacecraft orbits of $98.77 \mathrm{~min}$, thus covering the $3.35 \mathrm{~h}$ occultation, together with substantial out-of-eclipse baseline. During the analysis of the occultation data, we obtained further observations of two transits of WASP-189 b with CHEOPS on 15 and 18 June 2020, which we subsequently included in the final analysis. The transit observations covered the transit, together with a total of six CHEOPS orbits obtained outside of it. The observations were interrupted for up to 41 and up to $17 \mathrm{~min}$ per orbit due to Earth occultations or passages through the South Atlantic Anomaly (SAA), respectively. These instances can be seen as gaps in the light curves displayed in Figs. 1 and 2. We used exposure times of $4.8 \mathrm{~s}$ and co-added, on board, seven individual exposures of the $G=6.55 \mathrm{mag}$ star, resulting in an effective cadence of $33.4 \mathrm{~s}$. A full description of the CHEOPS telescope and the technical details of its observations is presented in Benz et al. (2020).

The data were processed with the CHEOPS data reduction pipeline (DRP, Hoyer et al. 2020), which performs image correction and uses aperture photometry to extract target fluxes for various apertures. The CHEOPS DRP was thoroughly tested, both using the CHEOPS data simulator (Futyan et al. 2020) and data obtained during commissioning. Using simulated data, we performed a series of injection and retrieval tests covering a range of planetary transit scenarios and levels of field crowding. The data obtained during the commissioning consisted of observations of stable stars that confirmed the stability of the photometry in the presence of interruptions due to SAA crossings and Earth occultations. During commissioning, we also 


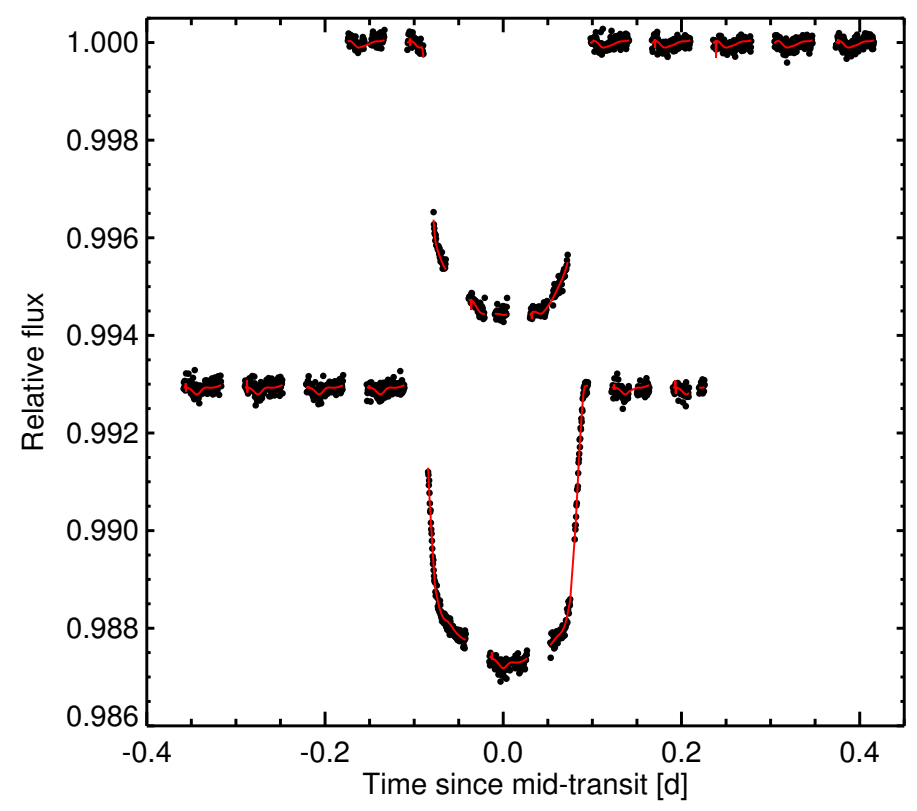

Fig. 2. Uncorrected CHEOPS observations of two transits of WASP$189 \mathrm{~b}$ (black points), together with their full (baseline and transit) lightcurve models (red lines). The upper light curve was observed on 15 June 2020 and the bottom light curve on 18 June 2020.

carried out transit observations and verified that the retrieved transit parameters were in good agreement with literature values (see e.g. Benz et al. 2020). For the occultations and the transits, versions 11 and 12 of the DRP were used, respectively. We found a minimal light curve RMS for the default aperture of 25 pixels.

Owing to the extended and irregular shape of CHEOPS' point spread function (PSF) and the fact that the field rotates around the target along the satellite's orbit, nearby stars produce a time-variable flux contamination in the photometric aperture, in phase with the spacecraft's roll angle. As explained in Hoyer et al. (2020), the DRP automatically determines the level of such contamination in the target's aperture for each exposure. The contamination is estimated from simulated images (Futyan et al. 2020) that are based on the CHEOPS PSF, the roll angle of each image. and the Gaia DR2 (Gaia Collaboration 2018) coordinates and magnitudes of all the stars with $G<19.5 \mathrm{mag}$ in the field of view. In order to determine the level of contamination, our simulations were created both with and without the target. Due to its brightness, WASP-189 appears to be well-isolated in the observed data, but the simulations show two faint contaminating sources located inside the aperture, with Gaia $\mathrm{G}$ magnitudes of 14.4 and 18.9 and distances of 9 and 19 arcsec from the target, respectively. Figure 3 shows a typical observation, as well as the corresponding simulated image containing only the background sources. We used these simulations to compute the time-variable contamination in the photometric aperture, finding that it is in excellent agreement with the observed flux variations on the CHEOPS orbital time scale. This allowed us to correct our photometric measurements for contamination (see Sect. 2.3).

\subsection{Host star properties}

To assist in our analysis of the WASP-189 system, we derived fundamental stellar parameters via spectral line and spectral energy distribution (SED) fitting, along with stellar evolution modelling. We estimated the stellar atmospheric parameters by comparing an average of 17 archival HARPS spectra with



Fig. 3. Example of the field of view of WASP-189 observed by CHEOPS (left) and its respective DRP simulation with the target removed (right). The circle and the cross represent the photometric aperture and the location of the target's PSF, respectively. The triangular shape of the CHEOPS PSF is clearly visible.

synthetic spectra computed using the synth 3 code (Kochukhov 2007), employing the tools described in Fossati et al. (2007). We computed stellar atmosphere models using LLmodels (Shulyak et al. 2004). We used an iterative procedure to derive the effective temperature ( $\left.T_{\text {eff }}\right)$ by imposing excitation equilibrium for both $57 \mathrm{FeI}$ and 10 FeII lines, the surface gravity $(\log g)$ by imposing $\mathrm{Fe}$ ionisation equilibrium, and the microturbulence velocity $\left(v_{\text {mic }}\right)$ by minimising the standard deviation in the $\mathrm{Fe}$ abundance. Prior to fitting the lines, we measured the stellar projected rotational velocity $\left(v \sin I_{*}=93.1 \pm 1.7 \mathrm{~km} \mathrm{~s}^{-1}\right)$ from several unblended lines. We confirmed this measurement by applying the Fourier analysis technique (Gray 2005; Murphy et al. 2016) to a handful of unblended lines. We find $T_{\text {eff }}=8000 \pm 80 \mathrm{~K}, \log g=3.9 \pm 0.2$, and $v_{\text {mic }}=2.7 \pm 0.3 \mathrm{~km} \mathrm{~s}^{-1}$. Both $T_{\text {eff }}$ and $\log g$ are in good agreement with those derived by Anderson et al. (2018). We measured an iron abundance $[\mathrm{Fe} / \mathrm{H}]$ of $+0.29 \pm 0.13 \mathrm{dex}$, as well as the abundances of $\mathrm{C}, \mathrm{O}, \mathrm{Na}, \mathrm{Mg}$, $\mathrm{Si}, \mathrm{S}, \mathrm{Ca}, \mathrm{Sc}, \mathrm{Ti}, \mathrm{Cr}, \mathrm{Ni}, \mathrm{Y}$, and $\mathrm{Ba}$, obtaining the pattern shown in Appendix A.

The derived abundance pattern is typical of chemically peculiar metallic-line (Am) stars (Fossati et al. 2007, 2008), which are limited to stars with a rotational velocity lower than $\approx 100 \mathrm{~km} \mathrm{~s}^{-1}$ (Michaud 1970). Therefore, as the measured stellar $v \sin I_{*}$ value is close to the maximum rotational velocity for which Am chemical peculiarities can arise, the stellar inclination angle should be close to $90^{\circ}$. The peculiar abundance pattern characterises only the stellar atmosphere and does not reflect the internal abundances, which we estimate at +0.2 dex from the abundances of $\mathrm{Mg}, \mathrm{Si}$, and $\mathrm{S}$ - elements that have been shown to be a good probe of the internal stellar metallicity (Fossati et al. 2007, 2008).

In order to determine the stellar radius of WASP-189, we utilised the infrared flux method (IRFM; Blackwell \& Shallis 1977), which permits the calculation of stellar angular diameter and $T_{\text {eff }}$ using previously derived relations between these parameters and optical and infrared broadband fluxes as well as the synthetic photometry conducted on stellar atmospheric models over the bandpasses of the observed data. We retrieved fluxes and corresponding uncertainties in the Gaia $\mathrm{G}, \mathrm{G}_{\mathrm{BP}}$, and $\mathrm{G}_{\mathrm{RP}}, 2 \mathrm{MASS} \mathrm{J}, \mathrm{H}$, and $\mathrm{K}$, and WISE $\mathrm{W} 1$ and $\mathrm{W} 2$ bandpasses taken from the most recent data release archives, respectively (Skrutskie et al. 2006; Wright et al. 2010; Gaia Collaboration 2018). Stellar synthetic models (Castelli \& Kurucz 2003) were fitted to the obtained broadband photometry in a Markov chain Monte Carlo (MCMC) approach, with priors on the stellar parameters taken from the spectroscopic analysis detailed 
above. The derived stellar angular diameter was combined with the Gaia parallax to determine the stellar radius, $R_{*, \mathrm{IRFM}}=$ $2.362 \pm 0.030 R_{\odot}$. This value is in good agreement with the value reported in the discovery paper (Anderson et al. 2018), with a precision, in fact, that is four times greater.

Finally, we used $T_{\text {eff }}$, metallicity (using $0.2 \pm 0.1 \mathrm{dex}$, see above), and $R_{*, \text { IRFM }}$ as inputs to obtain stellar mass and age through stellar evolution modelling. We merged the results from two independent approaches and stellar evolution codes: the Liège code CLES with a Levenberg-Marquardt approach, as in Buldgen et al. (2016), and the PARSEC code with the approach described in Bonfanti et al. (2015, 2016). We varied the input physics in stellar models (particularly with regard to the importance of convective overshooting and mixing of elements induced by diffusion) and we checked the consistency between our two approaches, which was found to be excellent. We ultimately infer a mass of $M_{*}=2.030 \pm 0.066 M_{\odot}$ and an age of $730 \pm 130$ Myr. The stellar parameters are listed in Table 1.

\subsection{CHEOPS data analysis}

We initially carried out an analysis that included only the occultations observed during the first weeks of scientific operations. However, later transit observations evidently showed an unexpectedly deep transit. We included these new data in our analysis, as a well-measured planetary radius is needed to properly interpret the occultation signal.

In addition to the astrophysical signals, the light curves contain the effect of variable contamination, which introduces a $\mathrm{V}$-shaped flux variation in phase with the spacecraft roll angle (clearly visible in Fig. 1). Furthermore, several visits show trends with time, the origin of which could lie in $\delta$ Scuti or $\gamma$ Doradus-type stellar pulsations.

\subsubsection{Occultation}

We carried out the analysis using an MCMC framework (CONAN, Lendl et al. 2020), modelling the occultation signal at the same time as these signals of non-planetary origin to ensure a full propagation of uncertainties. To account for correlated noise, we made use of either parametric models (e.g. Gillon et al. 2010) or Gaussian Processes (GP; using the George package Ambikasaran et al. 2016), or a combination of both (i.e. using a parametric function multiplied with the transit model as the GP mean model). To prescribe the occultation light curve, we used a limb-darkening-free Mandel \& Agol (2002) transit model. To account for our knowledge of the planetary transit parameters, we placed Gaussian priors corresponding to the values and uncertainties found from the CHEOPS transits (see Sect. 2.3.2) on the impact parameter, $b$, and the transit duration, $T_{14}$, the radius ratio, $R_{\mathrm{P}} / R_{*}$. Uniform priors were assumed for the occultation depth, $d F_{\text {occ }}$, and the mid-transit time, $T_{0}$. The period was kept fixed and the eccentricity was assumed to be zero (as found by Anderson et al. 2018). For the radial velocity amplitude, $K$, and the stellar mass and radius $\left(M_{*}, R_{*}\right)$, which are unconstrained by our analysis, we assumed Gaussian distributions, centred on the values of Anderson et al. (2018) or, where appropriate, the values reported in Sect. 2.2.

We explored a large range of models for the correlated noise, testing both parametric models composed of polynomials up to 4th order in the recorded state variables (most importantly: time, PSF centre, contamination, and spacecraft roll angle) as well as GPs using time, roll angle, and contamination, or a combination of these, as input. We tested both a Matérn-3/2

Table 1. Summary of stellar, input, and derived parameters of the WASP-189 system.

\begin{tabular}{|c|c|}
\hline \multicolumn{2}{|l|}{ Fitted parameters } \\
\hline $\begin{array}{l}\text { Mid-transit time }\left(T_{0}\right) \\
{\left[\mathrm{BJD}_{\mathrm{TT}}-2450000\right]}\end{array}$ & $8926.5416960_{-0.000064}^{+0.000065}$ \\
\hline Impact parameter $(b)$ & $0.478_{-0.012}^{+0.009}$ \\
\hline Scaled semi-major axis $\left(a / R_{*}\right)$ & $4.60_{-0.025}^{+0.031}$ \\
\hline Eclipse duration $\left(T_{14}\right)[\mathrm{h}]$ & $4.3336_{-0.0058}^{+0.0054}$ \\
\hline Occultation depth $\left(\mathrm{d} F_{\text {occ }}\right)[\mathrm{ppm}]$ & $87.9 \pm 4.3$ \\
\hline Radius ratio $\left(R_{\mathrm{p}} / R_{*}\right)$ & $0.07045_{-0.00015}^{+0.00013}$ \\
\hline$u_{+}=u_{a}+u_{b}$ & $0.550_{-0.017}^{+0.016}$ \\
\hline$u_{-}=u_{a}-u_{b}$ & $0.440_{-0.065}^{+0.066}$ \\
\hline Stellar inclination $I_{*}\left[{ }^{\circ}\right]$ & $75.5_{-2.2}^{+3.1}$ \\
\hline Projected orbital obliquity $\lambda\left[{ }^{\circ}\right]$ & $86.4_{-4.4}^{+2.9}$ \\
\hline \multicolumn{2}{|l|}{ Additional input parameters } \\
\hline $\mathrm{RV}$ amplitude $(K)\left[\mathrm{km} \mathrm{s}^{-1}\right]$ & $0.182 \pm 0.013$ \\
\hline Planetary period $^{(a)}(P)[\mathrm{d}]$ & 2.7240330 \\
\hline Eccentricity $^{(a)}(e)$ & 0 \\
\hline
\end{tabular}

Stellar parameters

Stellar Mass $\left(M_{*}\right)\left[M_{\odot}\right]$

$2.030 \pm 0.066$

Stellar Radius $\left(R_{*}\right)\left[R_{\odot}\right]$

$2.36 \pm 0.030$

Stellar eff. temperature $\left(T_{\text {eff }}\right)[\mathrm{K}]$

$8000 \pm 80$

Stellar surface gravity $\log g[\log g]$

$3.9 \pm 0.2$

Projected rotational velocity

$93.1 \pm 1.7$

$\nu \sin I_{*}\left[\mathrm{~km} \mathrm{~s}^{-1}\right]$

Microturbulent velocity $\quad 2.7 \pm 0.3$

$v_{\text {mic }}\left[\mathrm{km} \mathrm{s}^{-1}\right]$

Iron abundance $[\mathrm{Fe} / \mathrm{H}]$

$+0.29 \pm 0.13$

System age [Myr]

$730 \pm 130$

Derived parameters

Plan. radius $\left(R_{\mathrm{P}}\right)\left[R_{J}\right]$

Plan. mass $\left(M_{\mathrm{P}}\right)\left[M_{J}\right]$

Plan. mean density $\left(\rho_{\mathrm{P}}\right)\left[\rho_{J}\right]$

Plan. surface gravity $\left(g_{\mathrm{P}}\right)\left[\mathrm{m} \mathrm{s}^{-2}\right]$

Orbital semi-major axis $(a)$ [au]

Orbital inclination $(i)\left[^{\circ}\right]$

True orbital obliquity $\Psi\left[^{\circ}\right]$

Dayside equilibrium temp. ${ }^{(b)}\left(T_{\text {eq }}\right)[\mathrm{K}]$

Brightness temp. ${ }^{(c)}\left(T_{\mathrm{b}}\right)[\mathrm{K}]$

Dayside temp. ${ }^{(d)}\left(T_{\text {day }}\right)[\mathrm{K}]$

$1.619 \pm 0.021$
$1.99_{-0.14}^{+0.16}$
$0.469_{-0.057}^{+0.0275}$
$18.8_{-1.8}^{+2.1}$
$0.05053 \pm 0.00098$
$84.03 \pm 0.14$
$85.4 \pm 4.3$
$3353_{-34}^{+27}$
$3348_{-35}^{+26}$
$3435 \pm 27$

Notes. ${ }^{(a)}$ Fixed. ${ }^{(b)} T_{\text {eq }}=T_{\text {eff }} \sqrt{R_{*} / a}\left(f\left(1-A_{\mathrm{B}}\right)\right)^{1 / 4}$, assuming immediate re-radiation $(f=2 / 3)$ and zero albedo $\left(A_{\mathrm{B}}=0\right) .{ }^{(c)}$ Assuming black body stellar and planetary SEDs. ${ }^{(d)}$ Assuming a PHOENIX stellar model spectrum, $A_{\mathrm{g}}=0$, and inefficient energy circulation $(\epsilon=0)$.

and an exponential-squared kernel. We find that the systematics are equally well-modelled by using either a combination of time polynomials (modelling the slow trends) paired with a Matérn-3/2 GP with the telescope roll angle as input (modelling the contamination), or a combination of first- and second-order time polynomials together with a linear dependence on the contamination value. Both the results and derived uncertainties associated with each approach are fully compatible. We selected the latter as our preferred model, as it accounts for our 


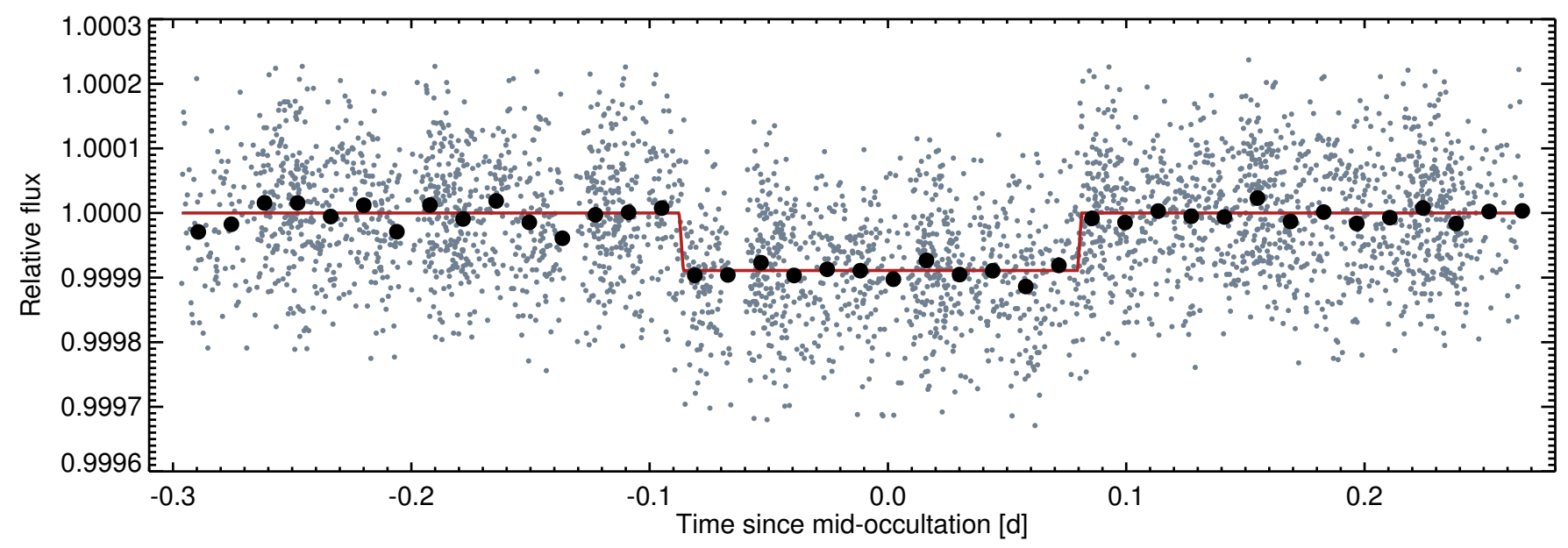

Fig. 4. Corrected and phase-folded CHEOPS occultation light curve of WASP-189 b. Black points show the light curve binned into 20-min intervals and the red line shows the final occultation model.

physical understanding of the source of the roll-angle-dependent variability. We report the results of our analysis in Table 1. Individual light curves are shown in Fig. 1, with the corrected and phase-folded data presented in Fig. 4.

We also carried out an independent analysis using the pycheops ${ }^{1}$ package, which is being developed specifically for the analysis of CHEOPS data. Optimisation of the model parameters was done using $1 \mathrm{mfit}^{2}$ and detrending done either via a parametric method of decorrelating the data linearly against the contamination or roll angle, and quadratically against time, or a GP regression with a Matérn-3/2 kernel to model the flux against roll angle trend using the celerite package (Foreman-Mackey et al. 2017). Again, we obtained values that are fully compatible with the reported ones.

\subsubsection{Transit}

At the photometric precision reached by CHEOPS, the planetary transit can be seen to be asymmetric, a feature most readily explained by the presence of gravity darkening due to the combination of the host star's fast rotation and the planet's inclined orbit (von Zeipel 1924; Barnes 2009). Accounting for gravity darkening in transit models is computationally intensive and, therefore, we performed an independent analysis of the transits and used the results as priors for the analysis of the occultations (see Sect. 2.3.1). We used the Transit and Light Curve Modeller (TLCM, see Csizmadia 2020 for details) for this purpose. This code uses the analytic expressions of Mandel \& Agol (2002) for the transit model and allows us to jointly model the transit together with various baseline models that account for correlated noise.

To model the gravity darkening, we compute a modification to the analytic model taking into account the varying stellar flux emitted along the planet's transit path. To do so, the stellar surface is divided into $120 \times 120$ surface elements (in longitude and in latitude) and, for each, the surface effective temperature is calculated via

$T_{\text {local }}=T_{*}\left(\frac{|\nabla V|_{\text {local }}}{|\nabla V|_{\text {pole }}}\right)^{0.25}$.

1 https://github.com/pmaxted/pycheops

2 https://lmfit.github.io/lmfit-py/
We assume a polar temperature of $T_{\text {pole }}=8000 \mathrm{~K}$ and the above equation inherently assumes a gravity darkening exponent of 1.0, which is appropriate for hot stars Claret et al. (2014). The local surface gravitational potential $(V)$ is calculated by assuming a two-axial ellipsoidal shape of the host star and given as $^{3}$

$V=\frac{n^{2} a^{3}}{(1+q) r}+\frac{1}{2} \omega_{\mathrm{rot}}^{2} r^{2} \sin ^{2} b$,

with the mass ratio, $q=M_{\mathrm{p}} / M_{*}$, the mean motion, $n$, and the astrographic latitude, $b$. The rotational angular velocity $\left(\omega_{\text {rot }}\right)$ is calculated from the stellar radius, $R_{\star}=2.36 \pm 0.030$, the $v \sin I_{*}=93.1 \pm 1.7 \mathrm{~km} \mathrm{~s}^{-1}$ (see Sect. 2.2), and the fitted stellar inclination. We fit two angles: the inclination of the stellar rotational vector, $I_{*}$, and its tilt-angle relative to celestial north direction $\left(\Omega_{\text {star }}=90^{\circ}-\lambda\right)$. These two angles fully describe the orientation of the stellar rotational axis. From the stellar and planetary orbital geometry and the stellar deformation, we infer the local stellar temperature behind the planetary disc. We then convert this temperature into a fractional light loss (or gain) compared to the nominal transit model, assuming black-body radiation and integrating over the CHEOPS' response function.

We fit these angles $\left(I_{*}, \Omega_{*}\right)$ together with the transit shape parameters, $R_{\mathrm{P}} / R_{*}, b, T_{0}$, the relative semi-major axis, $a / R_{*}$, and the linear combinations of the quadratic limb-darkening coefficients, $u_{+}=u_{a}+u_{b}$ and $u_{-}=u_{a}-u_{b}$. We assume a circular orbit and fix the period to that measured by Anderson et al. (2018). The roll-angle-dependent flux variation is accounted for through a baseline model in form of a fourth-order Fourier series for each light curve and we allow for a constant normalisation offset. As described in Csizmadia (2020), we first explored a wide parameter space using a series of genetic algorithm and simulated annealing chains, before using the best solution found as a starting point for five independent MCMC chains of $10^{6}$ steps each. The convergence was checked through the Gelman \& Rubin (1992) statistic.

We find a projected stellar obliquity of $\lambda=86.4_{-4.4}^{+2.9^{\circ}}$. The true obliquity $\Psi$ - the angle between the stellar rotational axis and the orbital angular momentum vector - can be calculated via

$\cos \Psi=\cos I_{*} \cos i+\sin I_{*} \sin i \cos \lambda$,

3 Stellar gravitational potential $V=G M / R_{*}$ was expressed by more easily measurable quantities via Kepler's third law. 


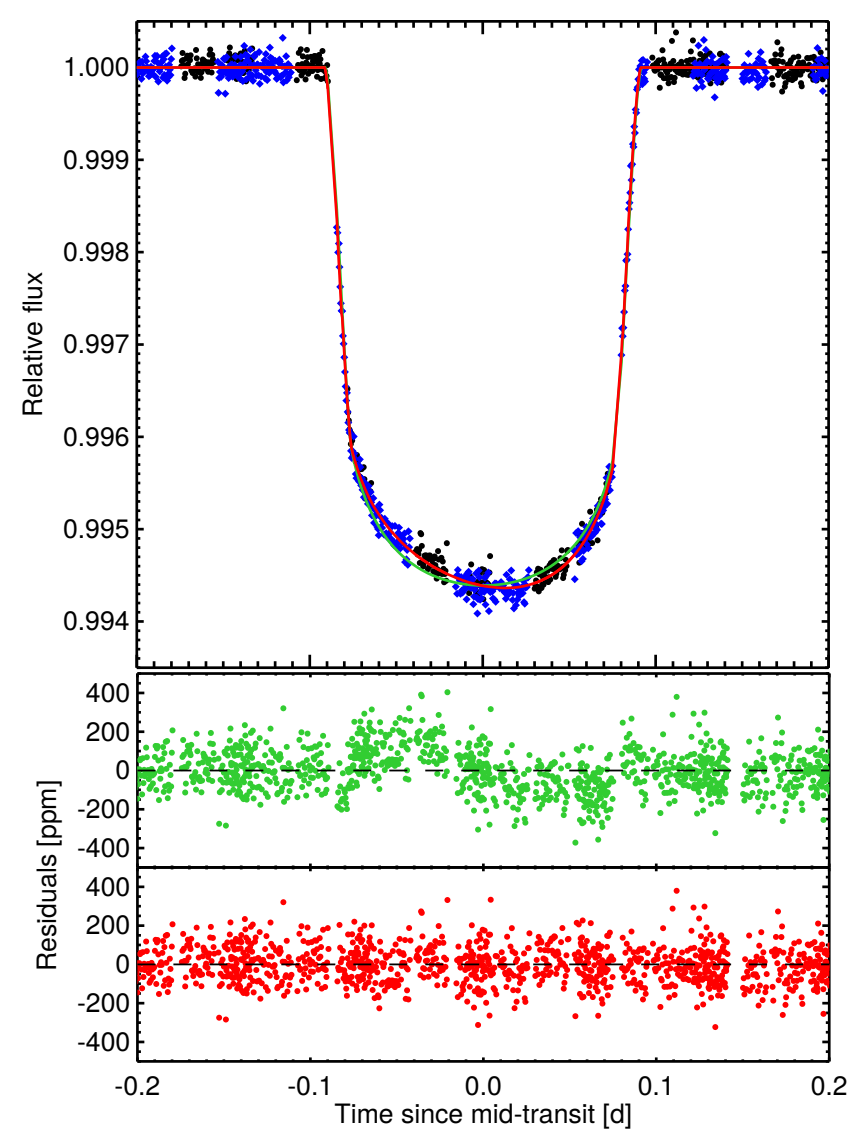

Fig. 5. Top: corrected and phase-folded transit light curve of WASP$189 \mathrm{~b}$. Data from the 15 June 2020 are shown as black circles and data from 18 June 2020 are shown as blue diamonds. The red and green curves illustrate the best-fit models, including and excluding gravity darkening, respectively. Bottom: data residuals related to each of the models. Green points in the upper panel refer to residuals in the model without gravity darkening and red points in the lower panel refer to that with gravity darkening.

and we find a value of $\Psi=85.4 \pm 4.3^{\circ}$. Here, $I_{*}$ and $i$ are the inclinations of the stellar rotational axis and the planetary orbit, respectively. The projected and true obliquity values found here are in good agreement with the findings of Anderson et al. (2018), who reported values of $\lambda=89.3 \pm 1.4^{\circ}$ and $\Psi=$ $90 \pm 5.8^{\circ}$ based on spectroscopic measurements.

We list all inferred and derived parameters in Table 1 . The full list of baseline function coefficients for transits and occultations is given in Appendix B. The individual and phase-folded transit light curves, together with the best-fit model, are shown in Figs. 2 and 5, respectively. For the sake of comparison, we also show a model fit obtained by assuming a spherical star without gravity darkening in Fig. 5 (green curve). It is evident from the residuals that the full model provides an improved fit for the asymmetric transit shape.

\section{Results}

\subsection{Revised planetary and system parameters}

The new, high-precision CHEOPS observations allow us to substantially revise the planetary parameters, and the gravitydarkened nature of the stellar photosphere allows us to derive an independent measurement of the projected angle between the stellar spin and the planetary orbital axes.
The remarkable difference of our results compared to those of Anderson et al. (2018) is that we find a $\sim 25 \%$ deeper transit, which is inconsistent with their published value at the level of $4.5 \sigma$. Paired with updated stellar parameters, this corresponds to a $\sim 15 \%$ larger planetary radius (inconsistent at $2.9 \sigma$ ) and, hence, a smaller planetary mean density. We attribute this discrepancy to the difficulties in obtaining high-precision photometry for bright stars from the ground given that the quality of ground-based data for bright stars is limited by the paucity of bright nearby reference stars. The photometric follow-up presented in Anderson et al. (2018) is, furthermore, limited to partial transits, which often suffer from imprecisely determined photometric trends that can bias the observed transit depth. In contrast, neither the time trends related to stellar variability nor the rollangle-dependent, in-orbit variations in CHEOPS data exhibit amplitudes that are large enough to create a transit depth offset of the observed magnitude. Furthermore, as described in Sect. 2.1, the CHEOPS DRS has been validated on well-known planetary transits.

From our gravity darkening analysis, we confirm a strongly misaligned orbit. While the analysis of the Rossiter-McLaughlin effect by Anderson et al. (2018) yields $\lambda=89.3 \pm 1.4^{\circ}$, our purely photometric analysis results in $\lambda=86.4_{-4.4}^{+2.9 \circ}$. Assuming that the star rotates more slowly than its break-up velocity, Anderson et al. (2018) find a true obliquity of $\Psi=90.0^{\circ} \pm 5.8^{\circ}$. Our photometric analysis is able to provide an assumption-free value of $\Psi=85.4^{\circ} \pm 4.3$.

\subsection{CHEOPS occultation measurement}

Based on a joint analysis of the four CHEOPS occultations, we determined the occultation depth of WASP-189 b in the CHEOPS passband to be $87.9 \pm 4.3 \mathrm{ppm}$. The precision of this measurement exceeds that of previous measurements obtained with CoRoT (Parviainen et al. 2013), and TESS (see Wong et al. 2020, and references therein), and is comparable in precision with the occultation depth measurements of hot Jupiters inferred from several quarters worth of Kepler data (e.g. Angerhausen et al. 2015; Esteves et al. 2015; Morris et al. 2013).

The individual, unbinned, occultation light curves, which have a cadence of $33.4 \mathrm{~s}$, have a residual RMS between 86 and $92 \mathrm{ppm}$. When applying binning into 10-min and 1-h intervals, we reach RMS values between 34 and 47, and 10 and $17 \mathrm{ppm}$, respectively. The phase-folded and binned residuals show an RMS of 23 and 5.7 ppm for 10-min and 1-h time bins, respectively. These values underline the excellent performance of CHEOPS.

Motivated by the high level of precision reached here, we also carried out independent analyses of each occultation to probe for any potential variation in the measured occultation depth. The occultation is detected at high significance in each individual light curve and the measurements are consistent at 1- $\sigma$ level. Thus, we find no significant sign of variability (see Table 2) in the dayside flux from WASP-189 b over the 19-day time span of our observations. At the same time, this illustrates that the value derived from a joint fit is not biased by any individual light curve.

\subsection{The atmosphere of WASP-189b}

\subsubsection{Model description}

To interpret the occultation depth, the radiative transfer code HELIOS was used to calculate the spectral energy distribution 

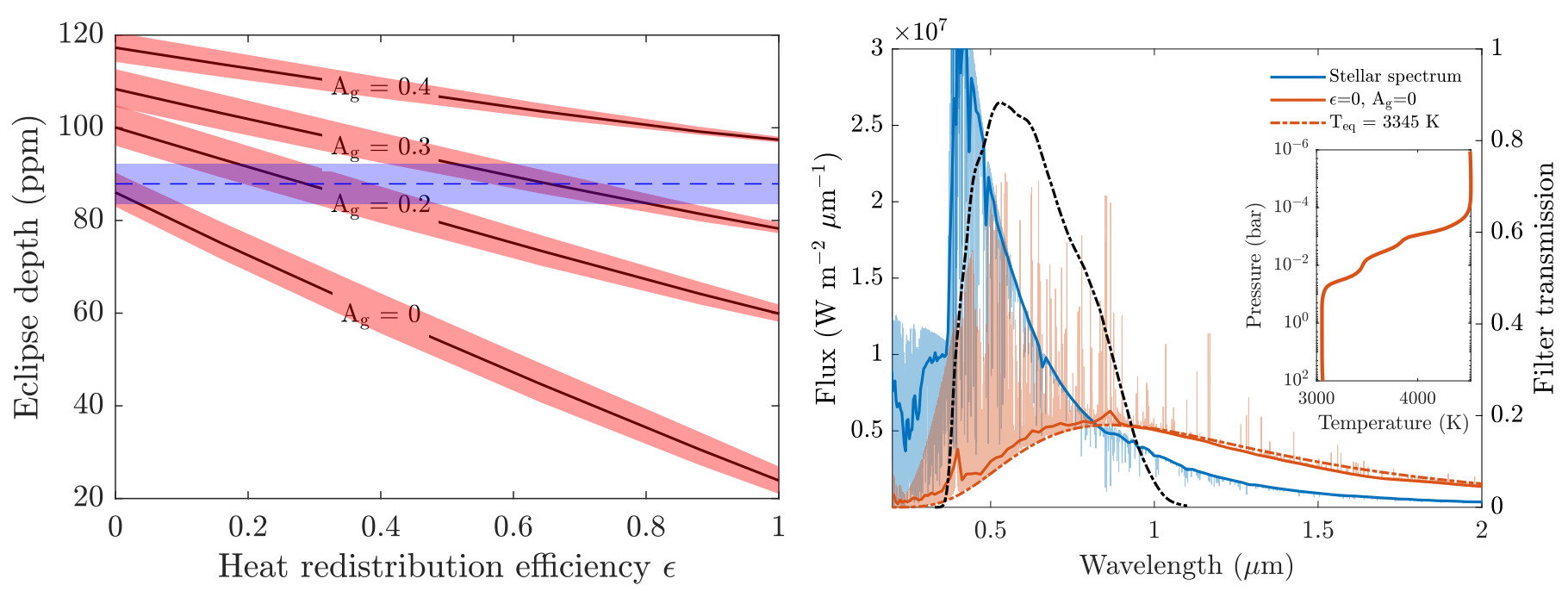

Fig. 6. Left panel: calculated (curves) versus measured (shaded horizontal region) occultation depths as functions of the heat redistribution efficiency. Different curves with assumed values of $A_{\mathrm{g}}$ are shown. As a sensitivity test, the shaded region associated with each curve corresponds to a variation in metallicity within a range of $[\mathrm{M} / \mathrm{H}]=0.2 \pm 0.3$. Right panel: theoretical spectral energy distribution, at a low and high resolution of the star (blue curve) with the CHEOPS bandpass (black dot-dashed curve) overlaid. The model for WASP-189 b (with $A_{\mathrm{g}}=\epsilon=0$ ) is overlaid in orange, with the corresponding temperature-pressure profile shown in the inset. For comparison, a blackbody curve with a temperature of $3345 \mathrm{~K}$ is also overlaid (orange dashed line).

Table 2. Occultation depths inferred from analyses of individual visits.

\begin{tabular}{lcccc}
\hline \hline Date (all 2020) & 19 March & 27 March & 30 March & 7 April \\
\hline $\mathrm{d} F_{\text {occ }}[\mathrm{ppm}]$ & $88.6_{-11}^{+8.5}$ & $83.5_{-8.5}^{+11.4}$ & $94.1_{-9.6}^{+9.9}$ & $89.3_{-6.9}^{+6.5}$ \\
\hline
\end{tabular}

(SED) of the dayside atmosphere of WASP-189 b. HELIOS solves for the thermal structure self-consistently (Malik et al. 2017, 2019). The model atmosphere is assumed to be cloud-free and in chemical equilibrium. We varied the planet's atmospheric metallicity within $[\mathrm{M} / \mathrm{H}]=0.2 \pm 0.3$, based on the stellar abundances. Sources of opacity include: spectral lines of atoms and ions of metals $\left(\mathrm{Ca}, \mathrm{Ca}^{+}, \mathrm{Fe}, \mathrm{Fe}^{+}, \mathrm{Ti}, \mathrm{Ti}^{+}, \mathrm{Na}, \mathrm{K}\right.$; Kurucz \& Bell 1995), which are predicted theoretically (e.g. Kitzmann et al. 2018) and observed at a high resolution in other ultra-hot Jupiters (e.g. Hoeijmakers et al. 2019); spectral lines of $\mathrm{H}_{2} \mathrm{O}, \mathrm{CO}, \mathrm{CH}_{4}$, VO and TiO (Barber et al. 2006; Yurchenko \& Tennyson 2014; Rothman et al. 2010; McKemmish et al. 2016, 2019); continuum absorption from the hydrogen anion $\left(\mathrm{H}^{-} ;\right.$John 1988$) ; \mathrm{H}_{2}-\mathrm{H}_{2}$, $\mathrm{H}_{2}-\mathrm{He}$ and $\mathrm{H}-\mathrm{He}$ collision-induced absorption (Karman et al. 2019). It is worth noting that HELIOS includes albedo contributions from Rayleigh scattering due to molecules. As illustrated in Fig. 6, our models predict that WASP-189 b possesses a thermal inversion, as inferred recently by Yan et al. (2020) from highresolution spectroscopic observations. We report the planetary dayside temperature in Table 1 , next to the brightness temperature computed under the assumption of black-body emission for star and planet. As described in Appendix C, these are discrepant because the assumption of black-body emission is flawed due to the proximity of the CHEOPS band to the Balmer jump.

The measured occultation depth can be explained by a combination of thermal emission and a weakly-reflective atmosphere (i.e. geometric albedo $\left.A_{\mathrm{g}} \sim[0.1-0.3]\right)$ for most values of the heat redistribution efficiency $(\epsilon$, see below). We note that thermal emission alone $\left(A_{\mathrm{g}}=0\right)$ may account for the measured occultation depth if zero heat redistribution is assumed $(\epsilon=0)$.

\subsubsection{Scattering by clouds/hazes}

Since the heat redistribution efficiency $(\epsilon)$ is unknown, a broader interpretation of the measured occultation depth may be obtained by assuming that scatterers of unknown origin and composition which are associated with clouds or hazes are present in the model atmosphere. They are parameterised by a single value of the geometric albedo $\left(A_{\mathrm{g}}\right)$. The occultation depth has contributions from reflected light and thermal emission, namely,

$\mathrm{d} F_{\text {occ }}=A_{\mathrm{g}}\left(\frac{R_{\mathrm{p}}}{a}\right)^{2}+\frac{\int \mathcal{F} F_{\mathrm{p}} \mathrm{d} \lambda}{\int \mathcal{F} F_{*} \mathrm{~d} \lambda}\left(\frac{R_{\mathrm{p}}}{R_{*}}\right)^{2}$.

The CHEOPS bandpass $(\mathcal{F})$, the SED of the star $\left(F_{*}\right.$, as computed in Sect. 2.2) and an example of the SED of WASP-189b $\left(F_{\mathrm{p}}\right)$ are shown in Fig. 6. As an input to HELIOS, the top-of-theatmosphere (TOA) flux impinging upon WASP-189 b is

$F_{\mathrm{TOA}}=F_{*}\left(\frac{R_{*}}{a}\right)^{2}\left(1-A_{\mathrm{B}}\right)\left(\frac{2}{3}-\frac{5 \epsilon}{12}\right)$,

where the heat redistribution efficiency $(0 \leq \epsilon \leq 1)$ follows the parametrisation of Cowan \& Agol (2011). It is related to the commonly used redistribution factor of $1 / 4 \leq f \leq 2 / 3$ (Seager et al. 2005) via $\epsilon=8 / 5-12 f / 5$. To relate the geometric and Bond $\left(A_{\mathrm{B}}\right)$ albedos, isotropic scattering is assumed such that $A_{\mathrm{g}}=2 A_{\mathrm{B}} / 3$.

Figure 6 shows that $A_{\mathrm{g}} \sim 0.1$ models are easily consistent with the measured occultation depth if $\epsilon \sim 0.1$, which is, in turn, consistent with the values of geometric albedos measured for cooler hot Jupiters (Heng \& Demory 2013).

\section{Conclusions and outlook}

In this paper, we present CHEOPS observations of the hot Jupiter WASP-189 b, capturing both the transit and the occultation of the highly irradiated planet. We robustly detect the occultation in individual epochs and measure a depth of $87.9 \pm 4.3 \mathrm{ppm}$ when 
combining four occultation light curves. Our measurement can be reproduced by atmospheric models with comparatively low albedo and heat redistribution efficiency. From two transit light curves, we derive updated planetary parameters and find a $\sim 15 \%$ larger planetary radius. The transits clearly show an asymmetric shape due to gravity darkening of the stellar host, and we use this effect to measure the planetary spin-orbit angle, finding a clearly misaligned orbit with a projected obliquity of $\lambda=86.4_{-4.4}^{+2.9 \circ}$ and a true obliquity of $\Psi=85.4 \pm 4.3^{\circ}$.

These observations showcase the capability of CHEOPS to detect shallow signals with an extremely high level of precision, thereby illustrating the potential of future studies of exoplanet atmospheres with CHEOPS. These will include (geometric) albedo measurements for cool planets, which have negligible contribution of thermal emission in the optical, as well as for planets, which have a dayside emission spectrum that is wellknown from infra-red observations. For the most favourable objects, CHEOPS will conduct phase curve observations, revealing the longitudinal cloud distribution in the planets' atmosphere. Thanks to its flexible pointing and observing schedule, CHEOPS can point to exoplanets across large areas of the sky, targeting the most rewarding objects. These practical aspects make CHEOPS an ideal facility for collecting a large sample of optical-light exoplanet occultations and phase curves.

Acknowledgements. CHEOPS is an ESA mission in partnership with Switzerland with important contributions to the payload and the ground segment from Austria, Belgium, France, Germany, Hungary, Italy, Portugal, Spain, Sweden, and the United Kingdom. The Swiss participation to CHEOPS has been supported by the Swiss Space Office (SSO) in the framework of the Prodex Programme and the Activités Nationales Complémentaires (ANC), the Universities of Bern and Geneva as well as well as of the NCCR PlanetS and the Swiss National Science Foundation. M.L.E. acknowledges support from the Austrian Research Promotion Agency (FFG) under project 859724 "GRAPPA". Sz. Cs. thanks DFG Research Unit 2440: 'Matter Under Planetary Interior Conditions: High Pressure, Planetary, and Plasma Physics' for support. Sz. Cs. acknowledges support by DFG grants RA 714/14-1 within the DFG Schwerpunkt SPP 1992 "Exploring the Diversity of Extrasolar Planets". A.D.E. and D.E.H. acknowledge support from the European Research Council (ERC) under the European Union's Horizon 2020 research and innovation programme (project FOUR ACES; grant agreement no. 724427). M.J.H. acknowledges the support of the Swiss National Fund under grant 200020_172746. The Spanish scientific participation in CHEOPS has been supported by the Spanish Ministry of Science and Innovation and the European Regional Development Fund through grants ESP2016-80435C2-1-R, ESP2016-80435-C2-2-R, ESP2017-87676-C5-1-R, PGC2018-098153B-C31, PGC2018-098153-B-C33, and MDM-2017-0737 Unidad de Excelencia María de Maeztu-Centro de Astrobiología (INTA-CSIC), as well as by the Generalitat de Catalunya/CERCA programme. The MOC activities have been supported by the ESA contract No. 4000124370. This work was supported by FCT - Fundação para a Ciência e a Tecnologia through national funds and by FEDER through COMPETE2020 - Programa Operacional Competitividade e Internacionalização by these grants: UID/FIS/04434/2019; UIDB/04434/2020; UIDP/04434/2020; PTDC/FIS-AST/32113/2017 \& POCI-01-0145-FEDER032113; PTDC/FIS-AST/28953/2017 \& POCI-01-0145-FEDER-028953; PTDC/FIS-AST/28987/2017 \& POCI-01-0145-FEDER-028987. S.C.C.B and S.G.S. acknowledge support from FCT through FCT contracts nr. IF/01312/2014/CP1215/CT0004, IF/00028/2014/CP1215/CT0002. O.D.S.D. is supported in the form of work contract (DL 57/2016/CP1364/CT0004) funded by national funds through Fundação para a Ciência e Tecnologia (FCT). The Belgian participation to CHEOPS has been supported by the Belgian Federal Science Policy Office (BELSPO) in the framework of the PRODEX Program, and by the University of Liege through an ARC grant for Concerted Research Actions financed by the Wallonia-Brussels Federation. M.G. is F.R.S.-FNRS Senior Research Associate. S.S. has received funding from the European Research Council (ERC) under the European Union's Horizon 2020 research and innovation programme (grant agreement No 833925, project STAREX) Gy.S. acknowledges funding from the Hungarian National Research, Development and Innovation Office (NKFIH) grant GINOP-2.3.2-15-2016-00003 and K-119517. For Italy, CHEOPS activities have been supported by the Italian Space Agency, under the programs: ASI-INAF n. 2013-016-R.0 and ASI-INAF n. 2019-29-HH.0. The team at LAM acknowledges CNES funding for the development of the CHEOPS DRP, including grants 124378 for O.D. and 837319 for S.H. X.B., S.C., D.G., M.F. and J.L. acknowledge their role as an ESA-appointed CHEOPS science team members.

\section{References}

Ambikasaran, S., Foreman-Mackey, D., Greengard, L., Hogg, D. W., \& O’Neil, M. 2016, IEEE Transactions on Pattern Analysis and Machine Intelligence, 38,252

Anderson, D. R., Temple, L. Y., Nielsen, L. D., et al. 2018, ArXiv e-prints [arXiv:1809.04897]

Angerhausen, D., DeLarme, E., \& Morse, J. A. 2015, PASP, 127, 1113

Asplund, M., Grevesse, N., Sauval, A. J., \& Scott, P. 2009, ARA\&A, 47, 481

Baglin, A., Auvergne, M., Boisnard, L., et al. 2006, 36th COSPAR Scientific Assembly, 3749

Barber, R. J., Tennyson, J., Harris, G. J., \& Tolchenov, R. N. 2006, Monthly Notices Roy. Astron. Soc., 368, 1087

Barnes, J. W. 2009, ApJ, 705, 683

Benz, W., Broeg, C., Fortier, A., et al. 2020, Exp. Astron., submitted [arXiv:2009.11633]

Blackwell, D. E., \& Shallis, M. J. 1977, MNRAS, 180, 177

Bonfanti, A., Ortolani, S., Piotto, G., \& Nascimbeni, V. 2015, A\&A, 575, A18

Bonfanti, A., Ortolani, S., \& Nascimbeni, V. 2016, A\&A, 585, A5

Borucki, W. J., Koch, D., Basri, G., et al. 2010, Science, 327, 977

Buldgen, G., Salmon, S. J. A. J., Reese, D. R., \& Dupret, M. A. 2016, A\&A, 596, A73

Castelli, F., \& Kurucz, R. L. 2003, in IAU Symp., 210, A20

Claret, A., Dragomir, D., \& Matthews, J. M. 2014, A\&A, 567, A3

Cowan, N. B., \& Agol, E. 2011, ApJ, 729, 54

Csizmadia, S. 2020, MNRAS, 496, 4442

Esteves, L. J., De Mooij, E. J. W., \& Jayawardhana, R. 2015, ApJ, 804, 150

Foreman-Mackey, D., Agol, E., Ambikasaran, S., \& Angus, R. 2017, AJ, 154 220

Fossati, L., Bagnulo, S., Monier, R., et al. 2007, A\&A, 476, 911

Fossati, L., Bagnulo, S., Landstreet, J., et al. 2008, A\&A, 483, 891

Futyan, D., Fortier, A., Beck, M., et al. 2020, A\&A, 635, A23

Gaia Collaboration (Brown, A. G. A., et al.) 2018, A\&A, 616, A1

Gaudi, B. S., Stassun, K. G., Collins, K. A., et al. 2017, Nature, 546, 514

Gelman, A., \& Rubin, D. 1992, Statist. Sci., 7, 457

Gillon, M., Lanotte, A. A., Barman, T., et al. 2010, A\&A, 511, A3

Gray, D. F. 2005, The Observation and Analysis of Stellar Photospheres (Cambridge, MA: Cambridge University Press)

Heng, K., \& Demory, B.-O. 2013, ApJ, 777, 100

Hoeijmakers, H. J., Ehrenreich, D., Kitzmann, D., et al. 2019, A\&A, 627, A165

Howell, S. B., Sobeck, C., Haas, M., et al. 2014, PASP, 126, 398

Hoyer, S., Guterman, P., Demangeon, O., et al. 2020, A\&A, 635, A24

Husser, T.-O., Wende-von Berg, S., Dreizler, S., et al. 2013, A\&A, 553, A6

John, T. L. 1988, A\&A, 193, 189

Karman, T., Gordon, I. E., van der Avoird, A., et al. 2019, Icarus, 328, 160

Kitzmann, D., Heng, K., Rimmer, P. B., et al. 2018, ApJ, 863, 183

Kochukhov, O. P. 2007, in Physics of Magnetic Stars, eds. I. I. Romanyuk, D. O. Kudryavtsev, O. M. Neizvestnaya, \& V. M. Shapoval, 109-118

Kurucz, R., \& Bell, B. 1995, in Atomic Line Data, eds. R.L. Kurucz, \& B. Bell, Kurucz CD-ROM No. 23, (Cambridge, MA: Smithsonian Astrophysical Observatory), 23

Lendl, M., Bouchy, F., Gill, S., et al. 2020, MNRAS, 492, 1761

Malik, M., Grosheintz, L., Mendonça, J. M., et al. 2017, AJ, 153, 56

Malik, M., Kitzmann, D., Mendonça, J. M., et al. 2019, AJ, 157, 170

Mandel, K., \& Agol, E. 2002, ApJ, 580, L171

McKemmish, L. K., Yurchenko, S. N., \& Tennyson, J. 2016, MNRAS, 463, 771

McKemmish, L. K., Masseron, T., Hoeijmakers, H. J., et al. 2019, MNRAS, 488, 2836

Michaud, G. 1970, ApJ, 160, 641

Morris, B. M., Mandell, A. M., \& Deming, D. 2013, ApJ, 764, L22

Murphy, S. J., Fossati, L., Bedding, T. R., et al. 2016, MNRAS, 459, 1201

Parviainen, H., Deeg, H. J., \& Belmonte, J. A. 2013, A\&A, 550, A67

Ricker, G. R., Winn, J. N., Vanderspek, R., et al. 2014, in Proc. SPIE, 9143, 914320

Rothman, L. S., Gordon, I. E., Barber, R. J., et al. 2010, J. Quant. Spec. Radiat. Transf., 111, 2139

Seager, S., Richardson, L. J., Hansen, B. M. S., et al. 2005, ApJ, 632, 1122

Shulyak, D., Tsymbal, V., Ryabchikova, T., Stütz, C., \& Weiss, W. W. 2004, A\&A, 428, 993

Skrutskie, M. F., Cutri, R. M., Stiening, R., et al. 2006, AJ, 131, 1163

von Zeipel, H. 1924, MNRAS, 84, 684 
Wong, I., Shporer, A., Daylan, T., et al. 2020, AJ, 160, 155

Wright, E. L., Eisenhardt, P. R. M., Mainzer, A. K., et al. 2010, AJ, 140, 1868

Yan, F., Pallé, E., Reiners, A., et al. 2020, A\&A, 640, L5

Yurchenko, S. N., \& Tennyson, J. 2014, MNRAS, 440, 1649

${ }^{1}$ Observatoire de Genève, Université de Genève, Chemin des maillettes 51, 1290 Sauverny, Switzerland

e-mail: Monika.Lendl@unige.ch

2 Space Research Institute, Austrian Academy of Sciences, Schmiedlstr. 6, 8042 Graz, Austria

3 Institute of Planetary Research, German Aerospace Center (DLR), Rutherfordstr. 2, 12489, Berlin, Germany

${ }^{4}$ Center for Space and Habitability, Gesellsschaftstr. 6, 3012 Bern, Switzerland

5 Laboratoire d'Astrophysique de Marseille, Univ. de Provence, UMR6110 CNRS, 38 r. F. Joliot Curie, 13388 Marseille, France

${ }^{6}$ Physikalisches Institut, University of Bern, Gesellschaftsstr. 6, 3012 Bern, Switzerland

7 Space sciences, Technologies and Astrophysics Research (STAR) Institute, Université de Liège, Allée du 6 Août 17, 4000 Liège, Belgium

8 Astrophysics Group, Cavendish Laboratory, J.J. Thomson Avenue, Cambridge CB3 OHE, UK

9 Astrophysics Group, Keele University, Staffordshire ST5 5BG, UK

${ }^{10}$ Department of Astronomy, Stockholm University, AlbaNova University Center, 10691 Stockholm, Sweden

11 School of Physics and Astronomy, Physical Science Building, North Haugh, St Andrews, UK

12 Astrobiology Research Unit, Université de Liège, Allée du 6 Août 19C, 4000 Liège, Belgium

13 Center for Astronomy and Astrophysics, Technical University Berlin, Hardenbergstr. 36, 10623 Berlin, Germany

${ }^{14}$ Instituto de Astrofísica de Canarias (IAC), 38200 La Laguna, Tenerife, Spain

15 Deptartamento de Astrofísica, Universidad de La Laguna (ULL), 38206 La Laguna, Tenerife, Spain

${ }^{16}$ ESTEC, European Space Agency, Keplerlaan 1, 2201 AZ Noordwijk, The Netherlands

${ }_{17}$ Admatis, Miskolc, Hungary

18 Depto. de Astrofísica, Centro de Astrobiología (CSIC-INTA), ESAC campus, 28692 Villanueva de la Cãda (Madrid), Spain

19 Instituto de Astrofísica e Ciências do Espaço, Universidade do Porto, CAUP, Rua das Estrelas, 4150-762 Porto, Portugal
${ }^{20}$ Departamento de Física e Astronomia, Faculdade de Ciências, Universidade do Porto, Rua do Campo Alegre, 4169-007 Porto, Portugal

${ }^{21}$ INAF, Osservatorio Astronomico di Padova, Vicolo dell'Osservatorio 5, 35122 Padova, Italy

${ }^{22}$ Université Grenoble Alpes, CNRS, IPAG, 38000 Grenoble, France

${ }^{23}$ Institut de Physique du Globe de Paris (IPGP), 1 rue Jussieu, 75005 Paris, France

${ }^{24}$ Lund Observatory, Department of Astronomy \& Theoretical Physics, Lund University, Box 43, Lund 22100, Sweden

${ }^{25}$ Leiden Observatory, University of Leiden, PO Box 9513, 2300 RA Leiden, The Netherlands

${ }^{26}$ Department of Space, Earth and Environment, Chalmers University of Technology, Onsala Space Observatory, 43992 Onsala, Sweden

27 INAF, Osservatorio Astrofisico di Torino, via Osservatorio 20, 10025 Pino Torinese, Italy

28 Division Technique INSU, BP 330, 83507 La Seyne cedex, France

${ }^{29}$ Konkoly Observatory, Research Centre for Astronomy and Earth Sciences, 1121 Budapest, Konkoly Thege Miklós út 15-17, Hungary

30 Institut d'astrophysique de Paris, UMR7095 CNRS, Université Pierre \& Marie Curie, 98bis blvd. Arago, 75014 Paris, France

${ }^{31}$ University of Vienna, Department of Astrophysics, Türkenschanzstr. 17, 1180 Vienna, Austria

32 IMCCE, UMR8028 CNRS, Observatoire de Paris, PSL Univ., Sorbonne Univ., 77 av. Denfert-Rochereau, 75014 Paris, France

33 INAF, Osservatorio Astrofisico di Catania, Via S. Sofia 78, 95123 Catania, Italy

${ }^{34}$ Institute of Optical Sensor Systems, German Aerospace Center (DLR), Rutherfordstr. 2, 12489 Berlin, Germany

35 Dipartimento di Fisica e Astronomia "Galileo Galilei", Universita' degli Studi di Padova, Vicolo dell'Osservatorio 3, 35122 Padova, Italy

36 Department of Physics, University of Warwick, Gibbet Hill Road, Coventry CV4 7AL, UK

37 Institut für Geologische Wissenschaften, Freie Universität Berlin, 12249 Berlin, Germany

38 Institut de Ciències de l'Espai (ICE, CSIC), Campus UAB, C/CanMagrans s/n, 08193 Bellaterra, Spain

39 Institut d'Estudis Espacials de Catalunya (IEEC), Gran Capità 2-4, 08034 Barcelona, Spain

40 ELTE Eötvös Loránd University, Gothard Astrophysical Observatory, Szombathely, Hungary

${ }^{41}$ MTA-ELTE Exoplanet Research Group, 9700 Szombathely, Szent Imre h. u. 112, Hungary

42 Institute of Astronomy, University of Cambridge, Madingley Road, Cambridge CB3 OHA, UK 
Appendix A: Stellar abundances

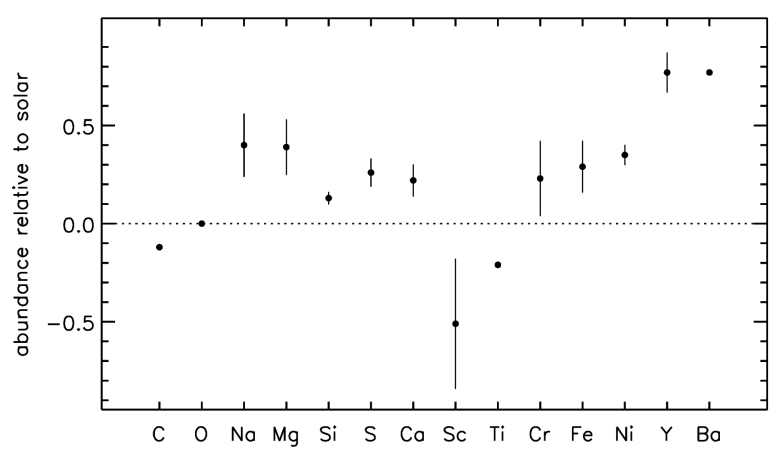

Fig. A.1. WASP-189 abundance pattern. The abundances are relative to solar (Asplund et al. 2009). The uncertainties are the standard deviation from the average abundance, therefore the abundances obtained from only one line $(\mathrm{C}, \mathrm{O}, \mathrm{Ti}, \mathrm{Ba})$ are shown without uncertainties.

The stellar abundance pattern is derived using the methods described in Sect. 2.2 and displayed in Fig. A.1.

\section{Appendix B: Photometric baseline model parameters}

In Table B.1, we report the inferred parameters and uncertainties for the baseline model parameters of each individual light curve.

Table B.1. Coefficients found for the photometric baselines models fitted jointly with the physical light curve model.

\begin{tabular}{|c|c|c|}
\hline \multicolumn{3}{|c|}{ Occultations } \\
\hline Date & 19 March 2020 & 27 March 2020 \\
\hline$\overline{A_{0}}$ & $1.0000805_{-0.0000093}^{+0.0000086}$ & $1.000024_{-0.0000096}^{+0.000010}$ \\
\hline$A_{1}$ & $-0.001221_{-0.000089}^{+0.000080}$ & $-0.001130_{-0.000101}^{0.000082}$ \\
\hline$A_{2}$ & $0.00162_{-0.00016}^{+0.00015}$ & $0.00184_{-0.00015}^{+0.00020}$ \\
\hline$D_{1}$ & $3.20_{-0.16}^{+0.17}$ & $2.22_{-0.18}^{+0.15}$ \\
\hline Date & 30 March 2020 & 07 April 2020 \\
\hline$A_{0}$ & $1.000033_{-0.000011}^{+0.000090}$ & $1.0000018_{-0.0000058}^{+0.0000066}$ \\
\hline$A_{1}$ & $-0.000090_{-0.000090}^{+0.000086}$ & $0.000189 \pm 0.000019$ \\
\hline$A_{2}$ & $0.00028_{-0.00015}^{0.00016}$ & 0 \\
\hline$D_{1}$ & $2.29 \pm 0.16$ & $1.95_{-0.16}^{+0.14}$ \\
\hline \multicolumn{3}{|c|}{ Transits } \\
\hline Date & 15 June 2020 & 18 June 2020 \\
\hline Flux shift (ppm) & \multicolumn{2}{|c|}{$-61 \pm 7$} \\
\hline$c_{1}(\mathrm{ppm})$ & $-76 \pm 14$ & $-30 \pm 14$ \\
\hline$c_{2}(\mathrm{ppm})$ & $+74 \pm 11$ & $-5 \pm 10$ \\
\hline$c_{3}(\mathrm{ppm})$ & $+78 \pm 16$ & $+23 \pm 17$ \\
\hline$c_{4}(\mathrm{ppm})$ & $+18 \pm 9$ & $+6 \pm 9$ \\
\hline$s_{1}(\mathrm{ppm})$ & $-40 \pm 14$ & $+90 \pm 11$ \\
\hline$s_{2}(\mathrm{ppm})$ & $-74 \pm 19$ & $+17 \pm 21$ \\
\hline$s_{3}(\mathrm{ppm})$ & $+3 \pm 11$ & $-13 \pm 11$ \\
\hline$s_{4}(\mathrm{ppm})$ & $+26 \pm 9$ & $-16 \pm 10$ \\
\hline
\end{tabular}

Notes. For the occultations, $A_{i}$ refer to the coefficients of second-order polynomials in time, with $A_{0}$ denoting the normalisation constant. $D_{1}$ is the coefficient of a linear trend with contamination. For the transits, $c$ stand for the cosine, and $s$ for the sine terms of the Fourier-series.

\section{Appendix C: Planetary brightness temperature}

We remark that, unlike the case of long-wavelength measurements, approximating the stellar emission by a black-body SED leads to an under-estimation of the stellar flux in the CHEOPS passband and, thus, it under-estimates the planetary dayside temperature. This is illustrated in Fig. C.1, which shows a model stellar spectrum compared to emission from an $8000 \mathrm{~K}$ black-body. The difference is attributed to the proximity of the CHEOPS band to the Balmer jump. For the case of WASP$189 \mathrm{~b}$, we find a brightness temperature of $3348_{-35}^{+26}$ when using the black-body approximation, but a higher value of $3435 \pm 27 \mathrm{~K}$ when using a stellar model spectrum.

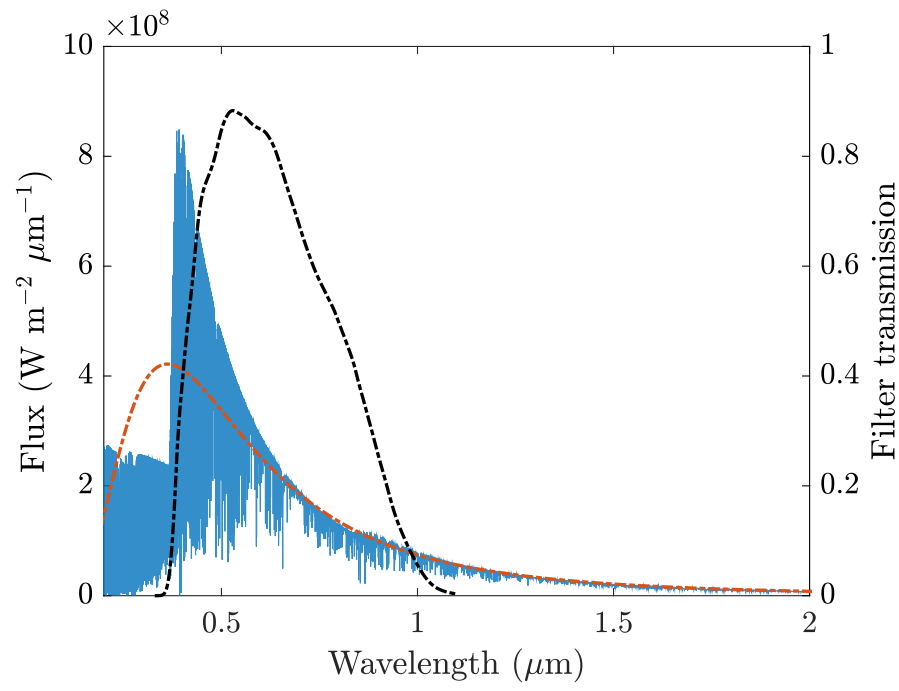

Fig. C.1. Comparison of a PHOENIX (Husser et al. 2013) stellar spectrum for a star with parameters corresponding to WASP-189 (blue), a $8000 \mathrm{~K}$ black-body (orange), and the CHEOPS passband (black). 